\title{
SCHUR-CONVEXITY OF A CLASS OF ELEMENTARY SYMMETRIC COMPOSITE FUNCTIONS
}

\author{
HUAN-NAN SHI ${ }^{1}$, TAO ZHANG ${ }^{2}$ \\ and BO-YAN XI ${ }^{3}$ \\ ${ }^{1}$ Department of Basic Teaching \\ Teacher's College \\ Beijing Union University \\ Beijing 100011 \\ P. R. China \\ e-mail: shihuannan2014@qq.com \\ sfthuannan@buu.edu.cn \\ ${ }^{2}$ School of Mathematical Sciences \\ Inner Mongolia University \\ Hohhot 010021 \\ P. R. China \\ e-mail: zhangtaocx@163.com
}

2020 Mathematics Subject Classification: 26E60, 26D16, 26A51.

Keywords and phrases: Schur-convesxity, Schur-geometric convexity, Schur-harmonic convexity, elementary symmetric function.

Received April 5, 2021

(C) 2021 Scientific Advances Publishers

This work is licensed under the Creative Commons Attribution International License (CC BY 3.0).

http://creativecommons.org/licenses/by/3.0/deed.en_US

Open Access (cc) (1)


${ }^{3}$ College of Mathematics

Inner Mongolia University for Nationalities

Tongliao City

Inner Mongolia Autonomous Region, 028043

P. R. China

e-mail: baoyintu78@qq.com

baoyintu68@sohu.com

baoyintu78@imun.edu.cn

\begin{abstract}
In this paper, using the properties of Schur-convex function, Schurgeometrically convex function and Schur-harmonically convex function, we provide much simpler proofs of the Schur-convexity, Schur-geometric convexity on $(1,+\infty)^{n}$, and Schur-harmonic convexity on $(1,+\infty)^{n}$ for a composite function of the elementary symmetric functions.
\end{abstract}

\title{
1. Introduction
}

Throughout the article, $\mathbb{R}$ denotes the set of real numbers, $\boldsymbol{x}=\left(x_{1}, x_{2}, \ldots, x_{n}\right)$ denotes $n$-tuple ( $n$-dimensional real vectors), the set of vectors can be written as

$$
\begin{aligned}
& \mathbb{R}^{n}=\left\{\boldsymbol{x}=\left(x_{1}, x_{2}, \ldots, x_{n}\right): x_{i} \in \mathbb{R}, i=1,2, \cdots, n\right\}, \\
& \mathbb{R}_{+}^{n}=\left\{\boldsymbol{x} \in \mathbb{R}^{n}: x_{i}>0, i=1,2, \cdots, n\right\}, \\
& \mathbb{R}_{-}^{n}=\left\{\boldsymbol{x} \in \mathbb{R}^{n}: x_{i}<0, i=1,2, \cdots, n\right\} .
\end{aligned}
$$

In particular, the notations $\mathbb{R}$ and $\mathbb{R}_{+}$denote $\mathbb{R}^{1}$ and $\mathbb{R}_{+}^{1}$, respectively.

Let $\boldsymbol{x}=\left(x_{1}, x_{2}, \cdots, x_{n}\right) \in \mathbb{R}^{n}$. The elementary symmetric functions are defined by

$$
E_{k}(\boldsymbol{x})=E_{k}\left(x_{1}, x_{2}, \cdots, x_{n}\right):=\sum_{1 \leq i_{1}<\cdots<i_{k} \leq n} \prod_{j=1}^{k} x_{i_{j}}, k=1, \cdots, n,
$$

$E_{0}(\boldsymbol{x})=1$ and $E_{k}(\boldsymbol{x})=0$ for $k<0$ or $k>n$. 
In 2006, Hua Mei et al. [7] studied the Schur-convexity of the following composite function of $E_{k}(\boldsymbol{x})$ :

$$
F_{k}(\boldsymbol{x})=E_{k}\left(\frac{1}{\boldsymbol{x}}-\boldsymbol{x}\right):=\sum_{1 \leq i_{1}<\cdots<i_{k} \leq n} \prod_{j=1}^{k}\left(\frac{1}{x_{i_{j}}}-x_{i_{j}}\right), k=1, \cdots, n .
$$

In 2012, using the Lemma 2.2 and Lemma 2.3 in second section, Shao [8] proved following the Theorem A and Theorem B, respectively.

Theorem A ([8]). (1) The function $E_{1}\left(\frac{1}{\boldsymbol{x}}-\boldsymbol{x}\right)$ is Schur-geometrically convex on $(0,1]^{n}, E_{n}\left(\frac{1}{\boldsymbol{x}}-\boldsymbol{x}\right)$ is Schur-geometrically concave on $(0,1]^{n}$.

(2) For $2 \leq k \leq n-1$ and $a=\frac{\sqrt{n-1}-\sqrt{k-1}}{\sqrt{n-k}}, E_{k}\left(\frac{1}{\boldsymbol{x}}-\boldsymbol{x}\right)$ is Schurgeometrically convex on $(0, a]^{n}$, and $E_{k}\left(\frac{1}{\boldsymbol{x}}-\boldsymbol{x}\right)$ is Schur-geometrically concave on $[a, 1]^{n}$.

Theorem B ([8]). For $k=1, \cdots, n, E_{k}\left(\frac{1}{\boldsymbol{x}}-\boldsymbol{x}\right)$ is Schur-harmonically concave on $(0,1]^{n}$.

The above results only relates to area $(0,1]^{n}$, in this paper, we study Schur-convexity and Schur-harmonic convexity of $E_{k}\left(\frac{1}{\boldsymbol{x}}-\boldsymbol{x}\right)$ on $\mathbb{R}^{n}$, and Schur-geometric convexity of $E_{k}\left(\frac{1}{\boldsymbol{x}}-\boldsymbol{x}\right)$ on $\mathbb{R}_{+}^{n}$, we prove the following results:

Theorem 1.1. Let $n \geq 2$.

(1) The function $E_{1}\left(\frac{1}{\boldsymbol{x}}-\boldsymbol{x}\right)$ is Schur-convex on $\mathbb{R}_{+}^{n}$, and $E_{1}\left(\frac{1}{\boldsymbol{x}}-\boldsymbol{x}\right)$ is Schur-concave on $\mathbb{R}_{-}^{n}$. 
(2) The function $E_{n}\left(\frac{1}{\boldsymbol{x}}-\boldsymbol{x}\right)$ is Schur-concave on $(-\infty,-1]^{n}$.

If $n$ is odd numbers (or even numbers), then $E_{n}\left(\frac{1}{\boldsymbol{x}}-\boldsymbol{x}\right)$ is Schurconvex (or Schur-concave) function on $(-1,-\sqrt{\sqrt{5}-2}]^{n}$ respectively.

If $n$ is odd numbers (or even numbers), then $E_{n}\left(\frac{1}{\boldsymbol{x}}-\boldsymbol{x}\right)$ is Schurconcave (or Schur-convex) function on $[-\sqrt{\sqrt{5}-2}, 0]^{n}$ respectively.

The function $E_{n}\left(\frac{1}{\boldsymbol{x}}-\boldsymbol{x}\right)$ is Schur-convex on $(0, \sqrt{\sqrt{5}-2}]^{n}$, and $E_{n}\left(\frac{1}{\boldsymbol{x}}-\boldsymbol{x}\right)$ is Schur-concave on $(\sqrt{\sqrt{5}-2}, 1]^{n}$.

If $n$ is odd numbers (or even numbers), then $E_{n}\left(\frac{1}{\boldsymbol{x}}-\boldsymbol{x}\right)$ is Schurconvex (or Schur-concave) function on $(1,+\infty)^{n}$ respectively.

(3) For $2 \leq k \leq n-1$, the $E_{k}\left(\frac{1}{\boldsymbol{x}}-\boldsymbol{x}\right)$ is Schur-concave on $(-\infty,-1]^{n}$.

If $k$ is odd numbers (or even numbers), then $E_{k}\left(\frac{1}{\boldsymbol{x}}-\boldsymbol{x}\right)$ is Schurconvex (or Schur-concave) function on $(-1,-\sqrt{\sqrt{5}-2}]^{n}$ respectively.

If $k$ is odd numbers (or even numbers), then $E_{k}\left(\frac{1}{\boldsymbol{x}}-\boldsymbol{x}\right)$ is Schurconcave (or Schur-convex) function on $[-\sqrt{\sqrt{5}-2}, 0]^{n}$ respectively.

The function $E_{k}\left(\frac{1}{\boldsymbol{x}}-\boldsymbol{x}\right)$ is Schur-convex on $[0, \sqrt{\sqrt{5}-2}]^{n}$.

If $n$ is odd numbers (or numbers even), then $E_{k}\left(\frac{1}{\boldsymbol{x}}-\boldsymbol{x}\right)$ is Schurconvex (or Schur-concave) function on $(1,+\infty)^{n}$ respectively. 
Theorem 1.2. For $k=1,2, \cdots, n$ with $n \geq 2$.

(1) The function $E_{1}\left(\frac{1}{\boldsymbol{x}}-\boldsymbol{x}\right)$ is Schur-geometrically concave on $[1,+\infty)^{n}$.

(2) If $n$ is odd numbers (or even numbers), then $E_{n}\left(\frac{1}{\boldsymbol{x}}-\boldsymbol{x}\right)$ is Schurgeometrically concave (or Schur-geometrically convex) function on $(1,+\infty)^{n}$ respectively.

(3) For $2 \leq k \leq n-1$, and $b=\frac{\sqrt{n-1}+\sqrt{k-1}}{\sqrt{n-k}}$, if $k$ is odd numbers (or even numbers), then $E_{k}\left(\frac{1}{\boldsymbol{x}}-\boldsymbol{x}\right)$ is Schur-geometrically convex (or Schur-geometrically concave) function on $[1, b]^{n}$ respectively, and $E_{k}\left(\frac{1}{\boldsymbol{x}}-\boldsymbol{x}\right)$ is Schur-geometrically concave (or Schur-geometrically convex) on $[b,+\infty)^{n}$.

Theorem 1.3. Let $n \geq 2$.

(1) The function $E_{1}\left(\frac{1}{\boldsymbol{x}}-\boldsymbol{x}\right)$ is Schur-harmonically convex on $\mathbb{R}_{+}^{n}$, and the $E_{1}\left(\frac{1}{\boldsymbol{x}}-\boldsymbol{x}\right)$ is Schur-harmonically concave on $\mathbb{R}_{-}^{n}$.

(2) The function $E_{n}\left(\frac{1}{\boldsymbol{x}}-\boldsymbol{x}\right)$ is Schur-harmonically convex on $(-\infty,-\sqrt{2+\sqrt{5}}]^{n}$.

The function $E_{n}\left(\frac{1}{\boldsymbol{x}}-\boldsymbol{x}\right)$ is Schur-harmonically concave on $[-\sqrt{2+\sqrt{5}},-1]^{n}$ 
If $n$ is odd numbers (or even numbers), then $E_{n}\left(\frac{1}{\boldsymbol{x}}-\boldsymbol{x}\right)$ is Schurharmonically convex (or Schur-harmonically concave) function on $[-1,0)^{n}$ respectively.

The $E_{n}\left(\frac{1}{\boldsymbol{x}}-\boldsymbol{x}\right)$ is Schur-harmonically concave on $(0,1]^{n}$.

If $n$ is odd numbers (or even numbers), then $E_{n}\left(\frac{1}{\boldsymbol{x}}-\boldsymbol{x}\right)$ is Schurharmonically convex (or Schur-harmonically concave) function on $[1, \sqrt{2+\sqrt{5}}]^{n}$ respectively.

If $n$ is odd numbers (or even numbers), then $E_{n}\left(\frac{1}{\boldsymbol{x}}-\boldsymbol{x}\right)$ is Schurharmonically concave (or convex) function on $[\sqrt{2+\sqrt{5}},+\infty)^{n}$ respectively.

(3) If $2 \leq k \leq n-1$, then the $E_{k}\left(\frac{1}{\boldsymbol{x}}-\boldsymbol{x}\right)$ is Schur-harmonically convex on $(-\infty,-\sqrt{2+\sqrt{5}}]^{n}$.

If $n$ is odd numbers (or even numbers), then $E_{n}\left(\frac{1}{\boldsymbol{x}}-\boldsymbol{x}\right)$ is Schurharmonically convex (or Schur-harmonically concave) function on $[-1,0)^{n}$ respectively.

The function $E_{k}\left(\frac{1}{\boldsymbol{x}}-\boldsymbol{x}\right)$ is Schur-harmonically concave on $(0,1]^{n}$.

If $n$ is odd numbers (or even numbers), then $E_{k}\left(\frac{1}{\boldsymbol{x}}-\boldsymbol{x}\right)$ is Schurharmonically concave (or Schur-harmonically convex) function on $[\sqrt{2+\sqrt{5}},+\infty)^{n}$ respectively. 


\section{Definitions and Lemmas}

For convenience, we recall some definitions as follows.

Definition 2.1. Let $\boldsymbol{x}=\left(x_{1}, x_{2}, \cdots, x_{n}\right)$ and $\boldsymbol{y}=\left(y_{1}, y_{2}, \cdots, y_{n}\right) \in \mathbb{R}^{n}$.

(1) $\boldsymbol{x} \geq \boldsymbol{y}$ means $x_{i} \geq y_{i}$ for all $i=1,2, \cdots, n$.

(2) Let $\Omega \subseteq \mathbb{R}^{n}, \varphi: \Omega \rightarrow \mathbb{R}$ is said to be increasing if $\boldsymbol{x} \geq \boldsymbol{y}$ implies $\varphi(x) \geq \varphi(y) . \varphi$ is said to be decreasing if and only if $-\varphi$ is increasing.

Definition $2.2([6,10])$. Let $\boldsymbol{x}=\left(x_{1}, x_{2}, \cdots, x_{n}\right)$ and $\boldsymbol{y}=\left(y_{1}, y_{2}, \cdots\right.$, $\left.y_{n}\right) \in \mathbb{R}^{n}$.

(1) $\boldsymbol{x}$ is said to be majorized by $\boldsymbol{y}$ (in symbols $\boldsymbol{x} \prec \boldsymbol{y}$ ) if $\sum_{i=1}^{k} x_{[i]} \leq \sum_{i=1}^{k} y_{[i]}$ for all $k=1,2, \cdots, n-1$ and $\sum_{i=1}^{n} x_{i}=\sum_{i=1}^{n} y_{i}$, where $x_{[1]} \geq x_{[2]} \geq \cdots \geq x_{[n]}$ and $y_{[1]} \geq y_{[2]} \geq \cdots \geq y_{[n]}$ are rearrangements of $\boldsymbol{x}$ and $\boldsymbol{y}$ in a descending order.

(2) $\Omega \subseteq \mathbb{R}^{n}$ is said to be a convex set if $\boldsymbol{x}, \boldsymbol{y} \in \Omega, 0 \leq \alpha \leq 1$, implies $\alpha \boldsymbol{x}+(1-\alpha) \boldsymbol{y}=\left(\alpha x_{1}+(1-\alpha) y_{1}, \alpha x_{2}+(1-\alpha) y_{2}, \cdots, \alpha x_{n}+(1-\alpha) y_{n}\right) \in \Omega$.

(3) Let $\Omega \subseteq \mathbb{R}^{n}$ be a symmetric and convex set, $\varphi: \Omega \rightarrow \mathbb{R}$ is said to be a Schur-convex function on $\Omega$ if $\boldsymbol{x} \prec \boldsymbol{y}$ on $\Omega$ implies $\varphi(\boldsymbol{x}) \leq \varphi(\boldsymbol{y})$. The function $\varphi$ is said to be Schur-concave on $\Omega$ if and only if $-\varphi$ is a Schur-convex function on $\Omega$.

Lemma 2.1 ([6, 10]) (Schur-convexity decision theorem). Let $\Omega \subseteq \mathbb{R}^{n}$ be a symmetric and convex set with nonempty interior $\Omega^{\circ}$. The function $\varphi: \Omega \rightarrow \mathbb{R}$ is continuous on $\Omega$ and continuously differentiable on $\Omega^{\circ}$. Then $\varphi$ is a Schur-convex (or Schur-concave, respectively) function if and only if $\varphi$ is symmetric on $\Omega$ and

$$
\left(x_{1}-x_{2}\right)\left(\frac{\partial \varphi}{\partial x_{1}}-\frac{\partial \varphi}{\partial x_{2}}\right) \geq 0 \text { (or } \leq 0, \text { respectively) }
$$

holds for any $\boldsymbol{x} \in \Omega^{\circ}$. 
The first systematical study of the functions preserving the ordering of majorization was made by Issai Schur in 1923. In Schur's honor, such functions are said to be "Schur-convex". It has many important applications in analytic inequalities, combinatorial optimization, quantum physics, information theory, and other related fields. See [6].

Definition 2.3 ([11]). Let $\boldsymbol{x}=\left(x_{1}, x_{2}, \cdots, x_{n}\right)$ and $\boldsymbol{y}=\left(y_{1}, y_{2}, \cdots, y_{n}\right)$ $\in \Omega \subseteq \mathbb{R}_{+}^{n}$.

(1) ([11, p. 64]) A set $\Omega$ is called a geometrically convex set if $\left(x_{1}^{\alpha} y_{1}^{\beta}, x_{2}^{\alpha} y_{2}^{\beta}, \cdots, x_{n}^{\alpha} y_{n}^{\beta}\right) \in \Omega$ for all $\boldsymbol{x}, \boldsymbol{y} \in \Omega$ and $\alpha, \beta \in[0,1]$ such that $\alpha+\beta=1$.

(2) ([11, p. 107]) Let $\Omega$ is a geometrically convex set. The function $\varphi: \Omega \rightarrow \mathbb{R}_{+}$is said to be a Schur-geometrically convex function on $\Omega$, for any $\quad \boldsymbol{x}, \boldsymbol{y} \in \Omega, \quad$ if $\quad\left(\ln x_{1}, \ln x_{2}, \cdots, \ln x_{n}\right) \prec\left(\ln y_{1}, \ln y_{2}, \cdots, \ln y_{n}\right)$ implies $\varphi(x) \leq \varphi(y)$. The function $\varphi$ is said to be a Schur-geometrically concave function on $\Omega$ if and only if $-\varphi$ is a Schur-geometrically convex function on $\Omega$.

Lemma 2.2 ([11, p. 108]) (Schur-geometrically convexity decision theorem). Let $\Omega \subseteq \mathbb{R}_{+}^{n}$ be a symmetric and geometrically convex set with a nonempty interior $\Omega^{\circ}$. Let $\varphi: \Omega \rightarrow \mathbb{R}_{+}$be continuous on $\Omega$ and differentiable in $\Omega^{\circ}$. If $\varphi$ is symmetric on $\Omega$ and

$$
\left(\ln x_{1}-\ln x_{2}\right)\left(x_{1} \frac{\partial \varphi}{\partial x_{1}}-x_{2} \frac{\partial \varphi}{\partial x_{2}}\right) \geq 0 \quad \text { (or } \leq 0, \text { respectively) }
$$

holds for any $\boldsymbol{x}=\left(x_{1}, x_{2}, \cdots, x_{n}\right) \in \Omega^{\circ}$, then $\varphi$ is a Schur-geometrically convex (or Schur-geometrically concave, respectively) function. 
The Schur-geometric convexity was proposed by Zhang [11] in 2004, and was investigated by Chu et al. [1], Guan [5], Sun et al. [9], and so on. We also note that some authors use the term "Schur multiplicative convexity".

In 2009, Chu ([4], [3], [2]) introduced the notion of Schurharmonically convex function.

Definition 2.4 ([4, 3, 2]). Let $\Omega \subseteq \mathbb{R}_{+}^{n}$ or $\Omega \subseteq \mathbb{R}_{-}^{n}, \boldsymbol{x}=\left(x_{1}, x_{2}, \cdots, x_{n}\right)$ and $\boldsymbol{y}=\left(y_{1}, y_{2}, \cdots, y_{n}\right) \in \Omega$.

(1) A set $\Omega$ is said to be harmonically convex if $\left(\frac{2 x_{1} y_{1}}{x_{1}+y_{1}}, \frac{2 x_{2} y_{2}}{x_{2}+y_{2}}, \cdots\right.$, $\left.\frac{2 x_{n} y_{n}}{x_{n}+y_{n}}\right) \in \Omega$ for every $\boldsymbol{x}, \boldsymbol{y} \in \Omega$

(2) Let $\Omega$ is a harmonically convex set, a function $\varphi: \Omega \rightarrow \mathbb{R}$ is said to be Schur-harmonically convex on $\Omega$, for any $\boldsymbol{x}, \boldsymbol{y} \in \Omega$, if $\left(\frac{1}{x_{1}}, \frac{1}{x_{2}}, \cdots, \frac{1}{x_{n}}\right) \prec\left(\frac{1}{y_{1}}, \frac{1}{y_{2}}, \cdots, \frac{1}{y_{n}}\right)$ implies $\varphi(x) \leq \varphi(y)$. A function $\varphi$ is said to be a Schur-harmonically concave function on $\Omega$ if and only if - $\varphi$ is a Schur-harmonically convex function on $\Omega$.

Lemma 2.3 ([4, 3, 2]) (Schur-harmonically convexity decision theorem). Let $\Omega \subseteq \mathbb{R}_{+}^{n}$ or $\Omega \subseteq \mathbb{R}_{-}^{n}$, be a symmetric and harmonically convex set with inner points and let $\varphi: \Omega \rightarrow \mathbb{R}$ be a continuous symmetric function which is differentiable on $\Omega^{\circ}$. Then $\varphi$ is Schur-harmonically convex (or Schur-harmonically concave, respectively) on $\Omega$ if and only if

$$
\left(x_{1}-x_{2}\right)\left(x_{1}^{2} \frac{\partial \varphi}{\partial x_{1}}-x_{2}^{2} \frac{\partial \varphi}{\partial x_{2}}\right) \geq 0 \text { (or } \leq 0 \text {, respectively), } \boldsymbol{x} \in \Omega^{\circ} .
$$


Remark 1. We extend the definition and determination theorem of Schur-harmonically convex function established by Chu as follows:

(1) $\Omega \subseteq \mathbb{R}_{+}^{n}$ is extended to $\Omega \subseteq \mathbb{R}_{+}^{n}$ or $\Omega \subseteq \mathbb{R}_{-}^{n}$.

(2) The function $\varphi: \Omega \rightarrow \mathbb{R}$ must not be a positive function.

Lemma 2.4 ([6, 10]). The function $E_{k}(\boldsymbol{x})$ is increasing and Schurconcave on $\mathbb{R}_{+}^{n}$. If $k>1, E_{k}(\boldsymbol{x})$ is strictly Schur-concave on $\mathbb{R}_{+}^{n}$.

Lemma 2.5. If $k$ is even numbers (or odd numbers, respectively), then $E_{k}(\boldsymbol{x})$ is decreasing and Schur-concave (or increasing and Schur-convex, respectively) on $\mathbb{R}_{-}^{n}$.

Proof. Notice that $E_{k}(-\boldsymbol{x})=(-1)^{k} E_{k}(\boldsymbol{x})$ for all $x \in \mathbb{R}_{+}^{n}$. Using the Lemma 2.4, it is easy to the desired result. Lemma 2.5 is proved.

It is easy to see that

Lemma 2.6. Let function $g(x)=\frac{x\left(1-x^{2}\right)}{1+x^{2}}$ for all $x \in \mathbb{R}$. Then

(1) the function $g$ is decreasing on $(-\infty,-\sqrt{\sqrt{5}-2}]$;

(2) the function $g$ is increasing on $[-\sqrt{\sqrt{5}-2},-\sqrt{\sqrt{5}-2}]$;

(3) the function $g$ is decreasing on $[\sqrt{\sqrt{5}-2},+\infty)$.

Lemma 2.7. Let function $g(x)=\frac{1-x^{2}}{x\left(1+x^{2}\right)}$ for all $x \in \mathbb{R} \backslash\{0\}$. Then

(1) the function $g$ is increasing on $(-\infty,-\sqrt{2+\sqrt{5}}]$ and $g$ is decreasing on $[-\sqrt{2+\sqrt{5}}, 0)$;

(2) the function $g$ is decreasing on $(0, \sqrt{2+\sqrt{5}}]$ and $g$ is increasing on $[\sqrt{2+\sqrt{5}},+\infty)$. 
It is easy to prove that the following lemma holds.

Lemma 2.8. Let function $h(x)=\frac{1}{x}-x$ for all $x \in \mathbb{R} \backslash\{0\}$. Then

(1) the function $h(x)>0$ for $x \in(-\infty,-1) \cup(0,1)$;

(2) the function $h(x)<0$ for $x \in(-1,0) \cup(1,+\infty)$.

\section{Proof of Theorems}

Proof of Theorem 1.1. For $k=1, \cdots, n, n \geq 2$, write

$$
\Delta_{k}(\boldsymbol{x})=\left(x_{1}-x_{2}\right)\left(\frac{\partial F_{k}(\boldsymbol{x})}{\partial x_{1}}-\frac{\partial F_{k}(\boldsymbol{x})}{\partial x_{2}}\right)
$$

The proof is divided into three cases.

(1) If $k=1$ and $\boldsymbol{x} \in \mathbb{R}_{+}^{n}$ (or $\boldsymbol{x} \in \mathbb{R}_{-}^{n}$, respectively), we have

$$
\Delta_{1}(\boldsymbol{x})=\frac{\left(x_{1}-x_{2}\right)^{2}\left(x_{1}+x_{2}\right)}{x_{1}^{2} x_{2}^{2}} \geq 0 \text { (or } \leq 0, \text { respectively) }
$$

By Lemma 2.1, it follows that Theorem 1.1(1) is holds. 
(2) If $k=n$ by Lemma 2.6 and Lemma 2.8, and notice that $\sqrt{\sqrt{5}-2}<1$, we have

$$
\begin{aligned}
& \Delta_{n}(\boldsymbol{x})= \frac{\left(x_{1}-x_{2}\right)\left(1+x_{1}^{2}\right)\left(1+x_{2}^{2}\right)}{x_{1}^{2} x_{2}^{2}}\left[\frac{x_{1}\left(1-x_{1}^{2}\right)}{1+x_{1}^{2}}-\frac{x_{2}\left(1-x_{2}^{2}\right)}{1+x_{2}^{2}}\right] F_{n-2}(\tilde{\boldsymbol{x}}) \\
& \geq 0, \quad \boldsymbol{x} \in(-1,-\sqrt{\sqrt{5}-2}]^{n}, \quad n \text { is odd number, } \\
& \leq 0, \quad \boldsymbol{x} \in(-\infty,-1]^{n}, \\
& \leq \begin{array}{ll}
\leq 0, & \boldsymbol{x} \in(-1,-\sqrt{\sqrt{5}-2}]^{n}, \quad n \text { is even number, } \\
\geq 0, & \boldsymbol{x} \in[-\sqrt{\sqrt{5}-2}, 0]^{n}, \quad n \text { is odd number, } \\
\geq 0, & \boldsymbol{x} \in(0,-\sqrt{\sqrt{5}-2}]^{n}, \\
\leq 0, & \boldsymbol{x} \in(\sqrt{\sqrt{5}-2}, 1]^{n}, \\
\geq 0, & \boldsymbol{x} \in(1,+\infty)^{n}, \quad n \text { is even number, } \\
\leq 0, & \boldsymbol{x} \in(1,+\infty)^{n}, \quad n \text { is even number, }
\end{array}
\end{aligned}
$$

where $\tilde{\boldsymbol{x}}=\left(x_{3}, \cdots, x_{n}\right)$.

By Lemma 2.1, it follows that Theorem 1.1 (2) is holds. 
(3) If $2 \leq k \leq n-1$ and $\boldsymbol{x} \in(0, \sqrt{\sqrt{5}-2}]^{n}$, by Lemma 2.6 and Lemma 2.8, and notice that $\sqrt{\sqrt{5}-2}<1$, we have

$$
\begin{aligned}
\Delta_{n}(\boldsymbol{x})= & \frac{\left(x_{1}-x_{2}\right)\left(1+x_{1}^{2}\right)\left(1+x_{2}^{2}\right)}{x_{1}^{2} x_{2}^{2}}\left[\frac{x_{1}\left(1-x_{1}^{2}\right)}{1+x_{1}^{2}}-\frac{x_{2}\left(1-x_{2}^{2}\right)}{1+x_{2}^{2}}\right] F_{k-2}(\tilde{\boldsymbol{x}}) \\
& +\frac{\left(x_{1}-x_{2}\right)^{2}\left(x_{1}+x_{2}\right)}{x_{1}^{2} x_{2}^{2}} F_{k-1}(\tilde{\boldsymbol{x}}) \\
= & \begin{cases}\leq 0, \quad \boldsymbol{x} \in(-\infty,-1]^{n}, \\
\geq 0, \quad \boldsymbol{x} \in(-1,-\sqrt{\sqrt{5}-2}]^{n}, \quad n \text { is odd number, } \\
\geq 0, \quad \boldsymbol{x} \in(-1,-\sqrt{\sqrt{5}-2}]^{n}, \quad n \text { is even number, } \\
\geq 0, \quad \boldsymbol{x} \in[-\sqrt{\sqrt{5}-2}, 0]^{n}, \quad n \text { is even number, } \\
\geq 0, \quad \boldsymbol{x} \in(0, \sqrt{\sqrt{5}-2}]^{n}, \\
\leq 0, \quad \boldsymbol{x} \in(1,+\infty)^{n}, \quad n \text { is odd number, }\end{cases}
\end{aligned}
$$

By the Lemma 2.1, it follows that Theorem 1.1(3) is holds.

The proof of Theorem 1.1 is completed.

Proof of Theorem 1.2. For $\boldsymbol{x}, \boldsymbol{y} \in[1,+\infty)^{n}$, if

$$
\left(\ln x_{1}, \ln x_{2}, \cdots, \ln x_{n}\right) \prec\left(\ln y_{1}, \ln y_{2}, \cdots, \ln y_{n}\right)
$$

implies

$\left(\ln \frac{1}{x_{1}}, \ln \frac{1}{x_{2}}, \cdots, \ln \frac{1}{x_{n}}\right) \prec\left(\ln \frac{1}{y_{1}}, \ln \frac{1}{y_{2}}, \cdots, \ln \frac{1}{y_{n}}\right)$ for $\frac{1}{\boldsymbol{x}}, \frac{1}{\boldsymbol{y}} \in(0,1]^{n}$.

Notice that $\frac{1}{b}=\frac{\sqrt{n-1}-\sqrt{k-1}}{\sqrt{n-k}}=a$, by Theorem A, this shows that 
(1) If $k=1$, we have

$$
-E_{1}\left(\frac{1}{\boldsymbol{x}}-\boldsymbol{x}\right)=E_{1}\left(\frac{1}{\frac{1}{\boldsymbol{x}}}-\frac{1}{\boldsymbol{x}}\right) \leq E_{1}\left(\frac{1}{\frac{1}{y}}-\frac{1}{\boldsymbol{y}}\right)=-E_{k}\left(\frac{1}{\boldsymbol{y}}-\boldsymbol{y}\right)
$$

and if $k=n$, then

$$
(-1)^{n} E_{n}\left(\frac{1}{\boldsymbol{x}}-\boldsymbol{x}\right)=E_{n}\left(\frac{1}{\frac{1}{\boldsymbol{x}}}-\frac{1}{\boldsymbol{x}}\right) \geq E_{n}\left(\frac{1}{\frac{1}{\boldsymbol{y}}}-\frac{1}{\boldsymbol{y}}\right)=(-1)^{n} E_{n}\left(\frac{1}{\boldsymbol{y}}-\boldsymbol{y}\right)
$$

(2) If $2 \leq k \leq n-1$ and $\boldsymbol{x}, \boldsymbol{y} \in[1, b]^{n}$, then

$$
(-1)^{k} E_{k}\left(\frac{1}{\boldsymbol{x}}-\boldsymbol{x}\right)=E_{k}\left(\frac{1}{\frac{1}{\boldsymbol{x}}}-\frac{1}{\boldsymbol{x}}\right) \geq E_{k}\left(\frac{1}{\frac{1}{\boldsymbol{y}}}-\frac{1}{\boldsymbol{y}}\right)=(-1)^{k} E_{k}\left(\frac{1}{\boldsymbol{y}}-\boldsymbol{y}\right)
$$

and if $2 \leq k \leq n-1$ and $\boldsymbol{x}, \boldsymbol{y} \in[b,+\infty)^{n}$, the above inequalities (3.2) is reversed.

By the Definition 2.4(2), from (1) and (2), it follows that Theorem 1.2 is holds.

Proof of Theorem 1.3. Let $\boldsymbol{x} \in \Omega \subseteq \mathbb{R}_{+}^{n}$ and $k=1, \cdots, n, n \geq 2$. Put

$$
\Lambda_{k}(\boldsymbol{x})=\left(x_{1}-x_{2}\right)\left(x_{1}^{2} \frac{\partial F_{k}(\boldsymbol{x})}{\partial x_{1}}-x_{2}^{2} \frac{\partial F_{k}(\boldsymbol{x})}{\partial x_{2}}\right)
$$

The proof is divided into three cases.

(1) If $k=1$, and $\boldsymbol{x} \in \mathbb{R}_{+}^{n}$ (or $\boldsymbol{x} \in \mathbb{R}_{-}^{n}$, respectively), we have

$$
\Delta_{1}(\boldsymbol{x})=-\left(x_{1}-x_{2}\right)^{2}\left(x_{1}+x_{2}\right) \leq 0 \text { (or } \leq 0 \text {, respectively). }
$$

By Lemma 2.3, it follows that Theorem 1.3(1) is holds. 
(2) If $k=n$, by Lemma 2.7 and Lemma 2.8, and notice that $\sqrt{\sqrt{5}+2}>2$, we get

$$
\begin{aligned}
\Lambda_{n}(\boldsymbol{x})= & \left(x_{1}-x_{2}\right)\left(1+x_{1}^{2}\right)\left(1+x_{2}^{2}\right)\left[\frac{1-x_{1}^{2}}{x_{1}\left(1+x_{1}^{2}\right)}-\frac{1-x_{2}^{2}}{x_{2}\left(1+x_{1}^{2}\right)}\right] F_{n-2}(\tilde{\boldsymbol{x}}) \\
\leq 0, & \boldsymbol{x} \in(-\infty,-\sqrt{2+\sqrt{5}}]^{n}, \\
\geq 0, & \boldsymbol{x} \in[-\sqrt{2+\sqrt{5}},-1]^{n}, \\
\leq 0, & \boldsymbol{x} \in[-1,0)^{n}, \quad n \text { is odd number, } \\
\leq 0, & \left.x \in[0,1]^{n}, 0\right)^{n}, \quad n \text { is even number, } \\
\geq 0, & \boldsymbol{x} \in[1, \sqrt{2+\sqrt{5}}]^{n}, \quad n \text { is odd number, } \\
\leq 0, & \boldsymbol{x} \in[1, \sqrt{2+\sqrt{5}}]^{n}, \quad n \text { is even number, } \\
\leq 0, & \boldsymbol{x} \in[\sqrt{2+\sqrt{5}},+\infty)^{n}, \quad n \text { is odd number, } \\
\geq 0, & \boldsymbol{x} \in[\sqrt{2+\sqrt{5}},+\infty)^{n}, \quad n \text { is even number. }
\end{aligned}
$$

By Lemma 2.3, it follows that Theorem 1.3(2) is holds. 
(3) If $2 \leq k \leq n-1$, by Lemma 2.7 and Lemma 2.8, and notice that $\sqrt{\sqrt{5}+2}>2$, we get

$$
\begin{aligned}
\Lambda_{k}(\boldsymbol{x})= & \left(x_{1}-x_{2}\right)\left(1+x_{1}^{2}\right)\left(1+x_{2}^{2}\right)\left[\frac{1-x_{1}^{2}}{x_{1}\left(1+x_{1}^{2}\right)}-\frac{1-x_{2}^{2}}{x_{2}\left(1+x_{2}^{2}\right)}\right] \\
& \times\left[F_{k-2}(\tilde{\boldsymbol{x}})\right]-\left(x_{1}-x_{2}\right)^{2}\left(x_{1}+x_{2}\right)\left[F_{k-1}(\tilde{\boldsymbol{x}})\right] \\
= & \begin{cases}\geq 0, \quad \boldsymbol{x} \in(-\infty,-\sqrt{2+\sqrt{5}}]^{n}, \\
\geq 0, \quad \boldsymbol{x} \in[-1,0)^{n}, \quad n \text { is odd number, } \\
\leq 0, \quad x \in[-1,0)^{n}, \quad n \text { is even number, } \\
\leq 0, \quad \boldsymbol{x} \in[\sqrt{2+\sqrt{5}},+\infty)^{n}, \quad n \text { is odd number, } \\
\geq 0, & \boldsymbol{x} \in[\sqrt{2+\sqrt{5}},+\infty)^{n}, \quad n \text { is even number. }\end{cases}
\end{aligned}
$$

By Lemma 2.3, it follows that Theorem 1.3(1) is holds.

The proof of Theorem 1.3 is completed.

\section{Applications}

Define

$$
A_{n}(\boldsymbol{x})=\frac{1}{n} \sum_{i=1}^{n} x_{i} \text { for } x \in \mathbb{R}^{n}, G_{n}(\boldsymbol{x})=\left(\prod_{i=1}^{n} x_{i}\right)^{\frac{1}{n}} \text { for } \boldsymbol{x} \in \mathbb{R}_{+}^{n}
$$

and

$$
H_{n}(\boldsymbol{x})=n\left(\sum_{i=1}^{n} \frac{1}{x_{i}}\right)^{-n} \text { for } \boldsymbol{x} \in \mathbb{R}_{+}^{n} \cup \mathbb{R}_{-}^{n}
$$

These means $A_{n}(\boldsymbol{x}), G_{n}(\boldsymbol{x})$, and $H_{n}(\boldsymbol{x})$ are respectively called the arithmetic, geometric, and harmonic means of numbers $x_{1}, x_{2}, \cdots, x_{n}$. 
Theorem 4.1. Let $n \geq 2$. If $\boldsymbol{x} \in(0, \sqrt{\sqrt{5}-2}]^{n}$ or $\boldsymbol{x} \in(-1,-\sqrt{\sqrt{5}-2}]^{n}$ $\cup(1,+\infty)^{n} \quad(2 \leq k \leq n$ and $k$ is odd number $)$ or $\boldsymbol{x} \in[-\sqrt{\sqrt{5}-2}, 0]^{n}$ $(2 \leq k \leq n$ and $k$ is even number $)$, or $\boldsymbol{x} \in \mathbb{R}_{+}^{n} \quad(k=1)$, then

$$
\left(\begin{array}{l}
n \\
k
\end{array}\right)\left(\frac{1}{A_{n}(\boldsymbol{x})}-A_{n}(\boldsymbol{x})\right)^{k} \leq E_{k}\left(\frac{1}{\boldsymbol{x}}-\boldsymbol{x}\right) .
$$

If $x \in(-\infty,-1]^{n}$ or $\boldsymbol{x} \in(-1,-\sqrt{\sqrt{5}-2}]^{n} \cup(1,+\infty)^{n} \quad(2 \leq k \leq n$ and $k$ is even number) or $\boldsymbol{x} \in[-\sqrt{\sqrt{5}-2}, 0]^{n}(2 \leq k \leq n$ and $k$ is odd number $)$, or $\boldsymbol{x} \in \mathbb{R}_{+}^{n} \quad(k=1)$, then the inequalities (4.3) is reversed.

Proof. For $\boldsymbol{x}=\left(x_{1}, x_{2}, \cdots, x_{n}\right) \in \mathbb{R}^{n}$, we have

$$
\left(A_{n}(\boldsymbol{x}), A_{n}(\boldsymbol{x}), \cdots, A_{n}(\boldsymbol{x})\right) \prec\left(x_{1}, x_{2}, \cdots, x_{n}\right)=\boldsymbol{x} .
$$

By Theorem 1.1 and Definition 2.2(3), the inequalities (4.3) holds. The proof is complete.

Theorem 4.2. Let $n \geq 2$, and let $a=\frac{\sqrt{n-1}-\sqrt{k-1}}{\sqrt{n-k}}$ and $b=\frac{\sqrt{n-1}+\sqrt{k-1}}{\sqrt{n-k}}$.

$$
\text { If } \boldsymbol{x} \in(0,1]^{n}(k=1) \text { or } \boldsymbol{x} \in(0, a]^{n}(2 \leq k \leq n-1) \text {, or } \boldsymbol{x} \in(1,+\infty)^{n}
$$

( $k=n$ and $k$ is even number), or $\boldsymbol{x} \in(1, b]^{n}(2 \leq k \leq n-1$ and $k$ is odd number), or $\boldsymbol{x} \in[b,+\infty)^{n}(2 \leq k \leq n-1$ and $k$ is even number $)$, then

$$
\left(\begin{array}{l}
n \\
k
\end{array}\right)\left(\frac{1}{G_{n}(\boldsymbol{x})}-G_{n}(\boldsymbol{x})\right)^{k} \leq E_{k}\left(\frac{1}{\boldsymbol{x}}-\boldsymbol{x}\right) .
$$


If $\boldsymbol{x} \in(0,1]^{n}(k=n)$ or and $\boldsymbol{x} \in(a, 1]^{n}(2 \leq k \leq n-1)$, or $\boldsymbol{x} \in(1,+\infty]^{n}$ $(k=1)$, or $\boldsymbol{x} \in(1,+\infty]^{n}(k=n$ and $k$ is odd number $)$, or $\boldsymbol{x} \in(1, b]^{n}(2 \leq$ $k \leq n-1$ and $k$ is even number), or $\boldsymbol{x} \in[b,+\infty)^{n}(2 \leq k \leq n-1$ and $k$ is odd number), then the inequalities (4.5) is reversed.

Proof. For all $\boldsymbol{x} \in \mathbb{R}_{+}^{n}$, we have

$$
\left(\ln G_{n}(\boldsymbol{x}), \ln G_{n}(\boldsymbol{x}), \cdots, \ln G_{n}(\boldsymbol{x})\right) \prec\left(\ln x_{1}, \ln x_{2}, \cdots, \ln x_{n}\right) .
$$

Form Definition 2.3(2), Theorem A and Theorem 1.2, we obtain the inequalities (4.5). Theorem 4.2 is proved.

Theorem 4.3. Let $n \geq 2$. If $\boldsymbol{x} \in \mathbb{R}_{+}^{n}(k=1)$, or $\boldsymbol{x} \in(-\infty,-\sqrt{2+\sqrt{5}}]^{n}$ $(2 \leq k \leq n)$, or $\boldsymbol{x} \in(-1,0]^{n} \quad(2 \leq k \leq n$ and $k$ is odd number $)$, or $\boldsymbol{x} \in(1, \sqrt{2+\sqrt{5}}]^{n}(k=n$ and $k$ is odd number $)$ or $\boldsymbol{x} \in(\sqrt{2+\sqrt{5}},+\infty)^{n}$ $(2 \leq k \leq n$ and $k$ is even number), then

$$
\left(\begin{array}{l}
n \\
k
\end{array}\right)\left(\frac{1}{H_{n}(\boldsymbol{x})}-H_{n}(\boldsymbol{x})\right)^{k} \leq E_{k}\left(\frac{1}{\boldsymbol{x}}-\boldsymbol{x}\right) .
$$

If $\boldsymbol{x} \in \mathbb{R}_{-}^{n}(k=1)$, or $\boldsymbol{x} \in[-\sqrt{2+\sqrt{5}},-1]^{n}(k=n)$, or $\boldsymbol{x} \in(-1,0]^{n}(2 \leq$ $k \leq n$ and $k \quad$ is even number $)$, or $\boldsymbol{x} \in(0,1]^{n}(2 \leq k \leq n)$ or $\boldsymbol{x} \in(1, \sqrt{2+\sqrt{5}}]^{n}(k=n$ and $k$ is even number $)$ or $\boldsymbol{x} \in(\sqrt{2+\sqrt{5}},+\infty)^{n}$ ( $2 \leq k \leq n$ and $k$ is odd number), then the inequalities (4.6) is reversed.

Proof. For $\boldsymbol{x} \in \mathbb{R}_{+}^{n} \cup \mathbb{R}_{-}^{n}$, we obtain

$$
\left(\frac{1}{H_{n}(\boldsymbol{x})}, \frac{1}{H_{n}(\boldsymbol{x})}, \cdots, \frac{1}{H_{n}(\boldsymbol{x})}\right) \prec\left(\frac{1}{x_{1}}, \frac{1}{x_{2}}, \cdots, \frac{1}{x_{n}}\right) .
$$

Using Definition 2.4(2), Theorem 1.3, the inequalities (4.6) holds. The proof is complete. 


\section{Acknowledgements}

This research was supported partially by the Inner Mongolia Normal University High-level Talent Research Startup Funding (2019YJRC010).

\section{References}

[1] Y.-M. Chu, X.-M. Zhang and G.-D. Wang, The Schur geometrical convexity of the extended mean values, Journal of Convex Analysis 15(4) (2008), 707-718.

[2] Y.-M. Chu and Y.-P. Lv, The Schur harmonic convexity of the Hamy symmetric function and its applications, Journal of Inequalities and Applications (2009); Article ID 838529, 10 pages.

DOI: https://doi.org/10.1155/2009/838529

[3] Y. M. Chu and T. C. Sun, The Schur harmonic convexity for a class of symmetric functions, Acta Mathematica Scientia 30(5) (2010), 1501-1506.

DOI: https://doi.org/10.1016/S0252-9602(10)60142-7

[4] Y.-M. Chu, G.-D.Wang and X.-H. Zhang, The Schur multiplicative and harmonic convexities of the complete symmetric function, Mathematische Nachrichten 284(5-6) (2011), 653-663.

DOI: https://doi.org/10.1002/mana.200810197

[5] K.-Z. Guan, Schur-convexity of the complete symmetric function, Mathematical Inequalities \& Applications 9(4) (2006), 567-576.

DOI: https://doi.org/10.7153/mia-09-52

[6] A. W. Marshall, I. Olkin and B. C. Arnold, Inequalities: Theory of Majorization and its Application, Second Edition, Springer, New York, 2011.

DOI: https://doi.org/10.1007/978-0-387-68276-1

[7] H. Mei, C.-L. Bai and H. Man, Extension of an Inequality Guess, Journal of Inner Mongolia University for Nationalities 21(2) (2006), 127-129. (in Chinese)

[8] Z.-H. Shao, The Schur-geometrical convexity and Schur-harmonic convexity for a class of symmetric functions, Mathematics in Practice and Theory 42(16) (2012), 199-206. (in Chinese)

[9] T.-C. Sun, Y.-P. Lv and Y.-M. Chu, Schur multiplicative and harmonic convexities of generalized Heronian mean in $n$ variables and their applications, International Journal of Pure and Applied Mathematics 55(1) (2009), 25-33.

[10] B.-Y. Wang, Foundations of Majorization Inequalities, Beijing Normal University Press, Beijing, 1990. (in Chinese)

[11] X.-M. Zhang, Geometrically Convex Functions, An'hui University Press, Hefei, 2004. (in Chinese) 